\title{
Hopf Bifurcation Analysis and Approximation of Limit Cycle in Coupled Van Der Pol and Duffing Oscillators
}

\author{
Jianping Cai ${ }^{*}$ a and Jianhe Shen ${ }^{\mathrm{b}}$ \\ ${ }^{a}$ Department of Mathematics and Information Science, Zhangzhou Normal University, Zhangzhou 363000, China \\ ${ }^{b}$ Department of Applied Mechanics and Engineering, Sun Yat-sen University, Guangzhou 510275, China
}

\begin{abstract}
Using Routh-Hurwitz criterion and Hopf bifurcation theorem, stability of the equilibrium points and Hopf bifurcation of coupled van der Pol and Duffing oscillators are investigated. The parametric curve corresponding to Hopf bifurcation and the existence region for the limit cycle in the parameter space are derived. The method of multiple time scales is used to deduce the analytical approximation of the limit cycle, and the accuracy of the analytical approximation is verified by direct numerical integration.
\end{abstract}

\section{INTRODUCTION}

Duffing oscillator and van der Pol oscillator are two classical nonlinear oscillators. Their dynamics are very rich in terms of stability, bifurcation and chaos. Since the last decade, much attention has been paid to the study of coupled oscillators, such as two coupled Duffing oscillators $[1,2]$ and two coupled van der Pol oscillators [3-5]. The reason for this study is twofold. On the one hand, various physical, electromechanical and biological systems can be described as a collection of coupled oscillators [6]. On the other hand, dynamical systems consisting of a collection of coupled oscillators are higher-dimensional and their dynamics is more complex and hence need further investigation. But, as pointed out by Chedjou et al. [7], less effort had been done in a system consisting of a self-excited oscillator and Duffing oscillator.

In this paper, a dynamical system consisting of coupled van der Pol and Duffing oscillators is discussed as follows

$$
\left\{\begin{array}{c}
x^{\prime \prime}+\omega_{1}^{2} x+\mu_{1}\left(1-x^{2}\right) x^{\prime}+f y^{\prime \prime}=0 \\
y^{\prime \prime}+\omega_{2}^{2} y+\mu_{2} y^{\prime}+c y^{3}-d x=0
\end{array}\right.
$$

where the prime denotes the derivative with respective to time and $\omega_{i}, \mu_{i}(i=1,2), c, d$ and $f$ are the parameters. Equations (1)-(2) can be used to model a self-sustained electromechanical transducer consisting of an electrical part and a mechanical part coupled through Laplace force and Lenz electromotive voltage, which was proposed by Chedjou et al. [7]. In the absence of the coupling, i.e., $f=d=0$, equations (1) and (2) reduce to the classical van der Pol oscillator and the damped Duffing oscillator without excitation respectively. Chedjou et al. [7] analyzed stability of the equilibriums of this equations using Routh-Hurwitz criterion and investigated the oscillatory state using the averaging method.

*Address correspondence to this author at the Department of Mathematics and Information Science, Zhangzhou Normal University, Zhangzhou 363000, China; E-mail: mathcai@hotmail.com
Shilnikov chaos of equations (1)-(2) was also studied by applying Shilnikov theorem. However, Hopf bifurcation and the parametric curve in the parametric space corresponding to Hopf bifurcation were not discussed in [7].

Our attention is addressed to Hopf bifurcation analysis of equations (1)-(2) and the existence and approximation of the limit cycle. The paper is arranged as follows. In section 2, stability of the equilibriums of equations (1)-(2) is analyzed. In section 3, Hopf bifurcation of equations (1)-(2) is analyzed and the parametric curve for Hopf bifurcation and the existence region for the limit cycle in the parameter space are obtained. In section 4, the method of multiple scales is used to deduce the analytical approximation for the limit cycle. The accuracy of the analytical approximation is compared with the results of direct numerical integration. The final section is devoted to some conclusions.

\section{STABILITY OF EQUILIBRIUM POINTS}

Equations (1)-(2) can be rewritten in the form

$$
\begin{aligned}
x_{1}^{\prime}= & x_{2}, \\
x_{2}^{\prime}= & -\mu_{1}\left(1-x_{1}^{2}\right) x_{2}-\left(\omega_{1}^{2}+f d\right) x_{1} \\
& +f \omega_{2}^{2} y_{1}+f c y_{1}^{3}+f \mu_{2} y_{2}, \\
y_{1}^{\prime}= & y_{2}, \\
y_{2}^{\prime}= & -\mu_{2} y_{2}-\omega_{2}^{2} y_{1}-c y_{1}^{3}+d x_{1} .
\end{aligned}
$$

From equations (3), it is easy to verify that

1. For $c \geq 0,\left(x_{10}, x_{20}, y_{10}, y_{20}\right)=(0,0,0,0)$ is the only equilibrium point of equations (1)-(2),

2. For $c<0$, besides $(0,0,0,0)$, $\left(x_{10}, x_{20}, y_{10}, y_{20}\right)=\left(0,0, \pm \frac{\omega_{2}}{\sqrt{-c}}, 0\right)$ are also the equilibrium points of equations (1)-(2). 
Linearizing equations (3) around a considered equilibrium point $\left(x_{10}, x_{20}, y_{10}, y_{20}\right)$ results in the following Jocabian matrix

$M=\left[\begin{array}{cccc}0 & 1 & 0 & 0 \\ -\left(2 \mu_{1} x_{10} x_{20}+\omega_{1}^{2}+f d\right) & \mu_{1}\left(1-x_{10}^{2}\right) & f\left(\omega_{2}^{2}+3 c y_{10}^{2}\right) & \mu_{2} f \\ 0 & 0 & 0 & 1 \\ d & 0 & -\left(\omega_{2}^{2}+3 c y_{10}^{2}\right) & -\mu_{2}\end{array}\right]$.

The eigenvalues of $M$ are the roots of the following characteristic polynomial

$\lambda^{4}+a_{1} \lambda^{3}+a_{2} \lambda^{2}+a_{3} \lambda+a_{4}=0$,

in which

$a_{1}=\mu_{2}-\mu_{1}\left(1-x_{10}^{2}\right)$,

$a_{2}=\omega_{1}^{2}+\omega_{2}^{2}+f d-\mu_{2} \mu_{1}\left(1-x_{10}^{2}\right)$

$+3 c y_{1}^{2}+2 \mu_{1} x_{10} x_{20}$,

$a_{3}=\mu_{2}\left(2 \mu_{1} x_{10} x_{20}+\omega_{1}^{2}\right)$

$-\mu_{1}\left(1-x_{10}^{2}\right)\left(\omega_{2}^{2}+3 c y_{10}\right)$,

$a_{4}=\left(\omega_{2}^{2}+3 c y_{10}^{2}\right)\left(\omega_{1}^{2}+2 \mu_{1} x_{10} x_{20}\right)$.

According to Routh-Hurwitz criterion, $M$ is a Hurwitz matrix and the considered equilibrium point $\left(x_{10}, x_{20}, y_{10}, y_{20}\right)$ is stable if the following inequalities hold,

$a_{i}(i=1,2,3,4)>0$,

$\Delta_{2}=a_{1} a_{2}-a_{3}>0$,

and

$\Delta_{3}=a_{1} a_{2} a_{3}-a_{3}^{2}-a_{1}^{2} a_{4}>0$.

\section{HOPF BIRURCATION ANALYSIS}

In what follows, the parameter $c \geq 0$ is pre-assumed, consequently, equation (1)-(2) process only an equilibrium point $(0,0,0,0)$. For the equilibrium point $(0,0,0,0)$, equations (6) reduce to

$a_{1}=\mu_{2}-\mu_{1}$,

$a_{2}=\omega_{1}^{2}+\omega_{2}^{2}+f d-\mu_{2} \mu_{1}$,

$a_{3}=\mu_{2} \omega_{1}^{2}-\mu_{1} \omega_{2}^{2}$,

$a_{4}=\omega_{2}^{2} \omega_{1}^{2}$.

Thus, according to Routh-Hurwitz criterion, the equilibrium point $(0,0,0,0)$ is stable if

$\mu_{2}>\mu_{1}$ and $\mu_{2} \mu_{1}<f d$.

Denote $a=f d-\mu_{2} \mu_{1}$ and suppose that $\omega_{1}=\omega_{2}$ such that the coupled oscillators (1)-(2) are in resonant state. Thus, for the equilibrium point $(0,0,0,0)$, equation (5) turns out to be

$$
\lambda^{4}+\left(\mu_{2}-\mu_{1}\right) \lambda^{3}+\left(2 \omega^{2}+a\right) \lambda^{2}+\left(\mu_{2}-\mu_{1}\right) \omega^{2} \lambda+\omega_{4}=0 .
$$

The characteristic roots of equation (9) is given by

$$
\begin{aligned}
& \lambda_{1,2}=-\frac{\mu_{2}-\mu_{1}}{4}+\frac{1}{2} \sqrt{-a+\frac{\left(\mu_{2}-\mu_{1}\right)^{2}}{4}} \pm \sqrt{\Delta_{1}}, \\
& \lambda_{3,4}=-\frac{\mu_{2}-\mu_{1}}{4}-\frac{1}{2} \sqrt{-a+\frac{\left(\mu_{2}-\mu_{1}\right)^{2}}{4}} \pm \sqrt{\Delta_{2}},
\end{aligned}
$$

where

$$
\Delta_{1}=\frac{1}{2}\left(\mu_{2}-\mu_{1}\right)^{2}-\frac{1}{2}\left(\mu_{2}-\mu_{1}\right) \sqrt{\left(\mu_{2}-\mu_{1}\right)^{2}-4 a}-a-4 \omega^{2},
$$

and

$$
\Delta_{2}=\frac{1}{2}\left(\mu_{2}-\mu_{1}\right)^{2}+\frac{1}{2}\left(\mu_{2}-\mu_{1}\right) \sqrt{\left(\mu_{2}-\mu_{1}\right)^{2}-4 a}-a-4 \omega^{2} .
$$

From equation (10a) we can find that

1) if $a=0$, i.e.

$\mu_{2} \mu_{1}=f d$,

Equation (10) turns out to be

$\lambda_{1,2}= \pm i \omega$ and $\lambda_{3,4}=-\frac{\mu_{2}-\mu_{1}}{2} \pm \sqrt{\left(\mu_{2}-\mu_{1}\right)^{2}-4 \omega^{2}}$.

2) if $a>0$, i.e.

$f d>\mu_{2} \mu_{1}$,

Equation (9) process two complex roots and two real roots with negative real parts.

3) if $a<0$, i.e.

$f d<\mu_{2} \mu_{1}$,

Equation (9) process two complex roots with positive real parts.

According to Hopf bifurcation theorem [8], under the parametric condition (12), Hopf bifurcation of equations (1)(2) occurs. Hence, equation (12) represents the parametric curve corresponding to Hopf bifurcation and inequality (13) determines the existence region of limit cycle in the parametric space.

Fix the parameters of equations (1)-(2) as follows: $\mu_{1}=0.33, d=0.66, f=0.33, c=0.01, \omega_{1}=\omega_{2}=1.0$. According to the discussions above, it can be concluded that for the control parameter $\mu_{2} \in(0.33,0.66)$, the equilibrium point $(0,0,0,0)$ is stable and $\mu_{2 c}=0.66$ is the critical parameter value corresponding to Hopf bifurcation. Direct numerical simulations shown in Figs. $(\mathbf{1 , 2})$ verify these conclusions.

\section{THE APPROXIMATION OF THE LIMIT CYCLE}

Next, we seek analytical approximation of the limit cycle nearing the equilibrium point by using the method of multiple scales. Supposing that the damp and the nonlinearity in the van der Pol oscillator and Duffing oscillator and the coupling between them are relatively small, equations (1)-(2) can be rescaled in the following form

$x^{\prime \prime}+\omega_{1}^{2} x+\varepsilon \mu_{1}\left(1-x^{2}\right) x^{\prime}+\varepsilon f y^{\prime \prime}=0$, 


$$
y^{\prime \prime}+\omega_{2}^{2} y+\varepsilon \mu_{2} y^{\prime}+\varepsilon c y^{3}-\varepsilon d x=0,
$$

where $\varepsilon$ formally stands for a dimensionless small parameter but it will be set to unit in the final analysis.
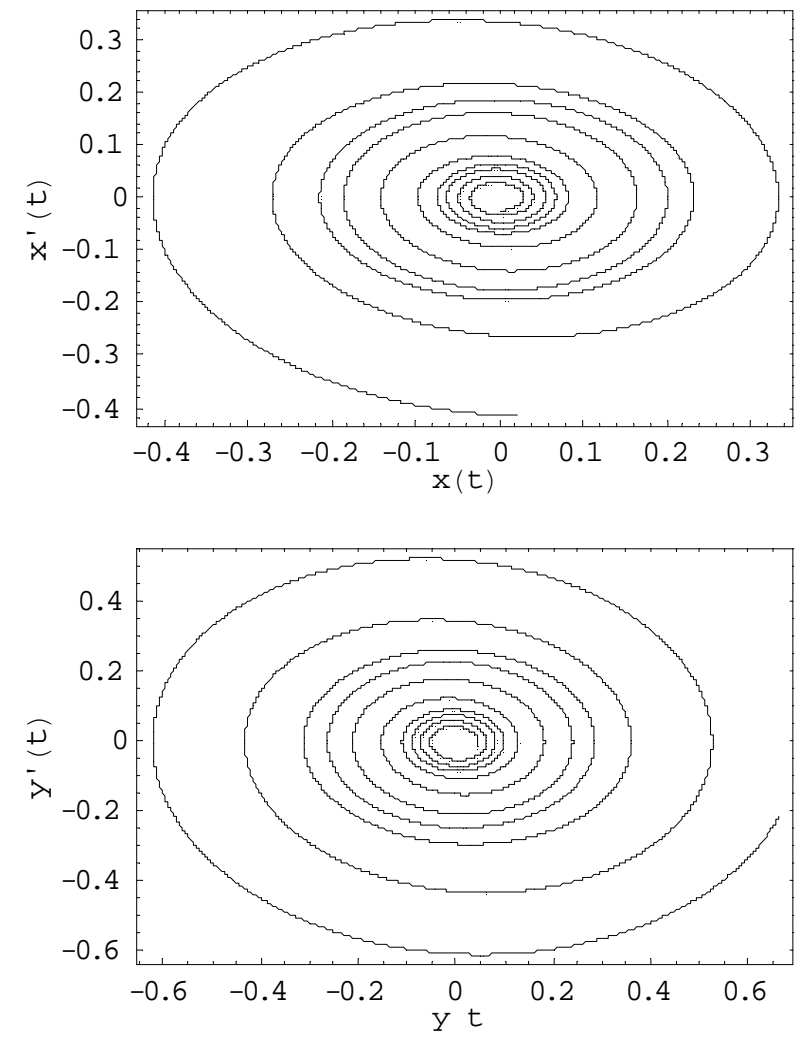

Fig. (1). Simulations of the orbit of equations (1)-(2) starting from an arbitrary initial condition when $\mu_{2}=0.5$.

We begin by introducing the time scales

$T_{0}=t, T_{1}=\varepsilon t, \cdots, T_{k}=\varepsilon^{k} t, \cdots, k=0,1,2 \cdots$,

which yields

$\frac{d}{d t}=D_{0}+\varepsilon D_{1}+\varepsilon^{2} D_{2}+\cdots$,

$\frac{d^{2}}{d t^{2}}=D_{0}^{2}+2 \varepsilon D_{0} D_{1}+\varepsilon^{2}\left(D_{1}^{2}+2 D_{0} D_{2}\right)+\cdots$.

Next, assume that the analytical formulation for the limit cycle is

$x(t, \varepsilon)=\sum_{k=0}^{\infty} \varepsilon^{k} x_{k}\left(T_{0}, T_{1}, T_{2}, \cdots\right)$,

$y(t, \varepsilon)=\sum_{k=0}^{\infty} \varepsilon^{k} y_{k}\left(T_{0}, T_{1}, T_{2}, \cdots\right)$.

Substituting equations (20)-(21) into equations (15)-(16) and balancing coefficient of the same power of $\varepsilon$ yields the ordered perturbation equations:

$\varepsilon^{0}: D_{0}^{2} x_{0}+\omega_{1}^{2} x_{0}=0$

$D_{0}^{2} y_{0}+\omega_{2}^{2} y_{0}=0$, $\varepsilon^{1}: D_{0}^{2} x_{1}+\omega_{1}^{2} x_{1}$

$=-2 D_{0} D_{1} x_{0}+\mu_{1}\left(1-x_{0}^{2}\right) D_{0} x_{0}+f \omega_{2}^{2} y_{0}$,
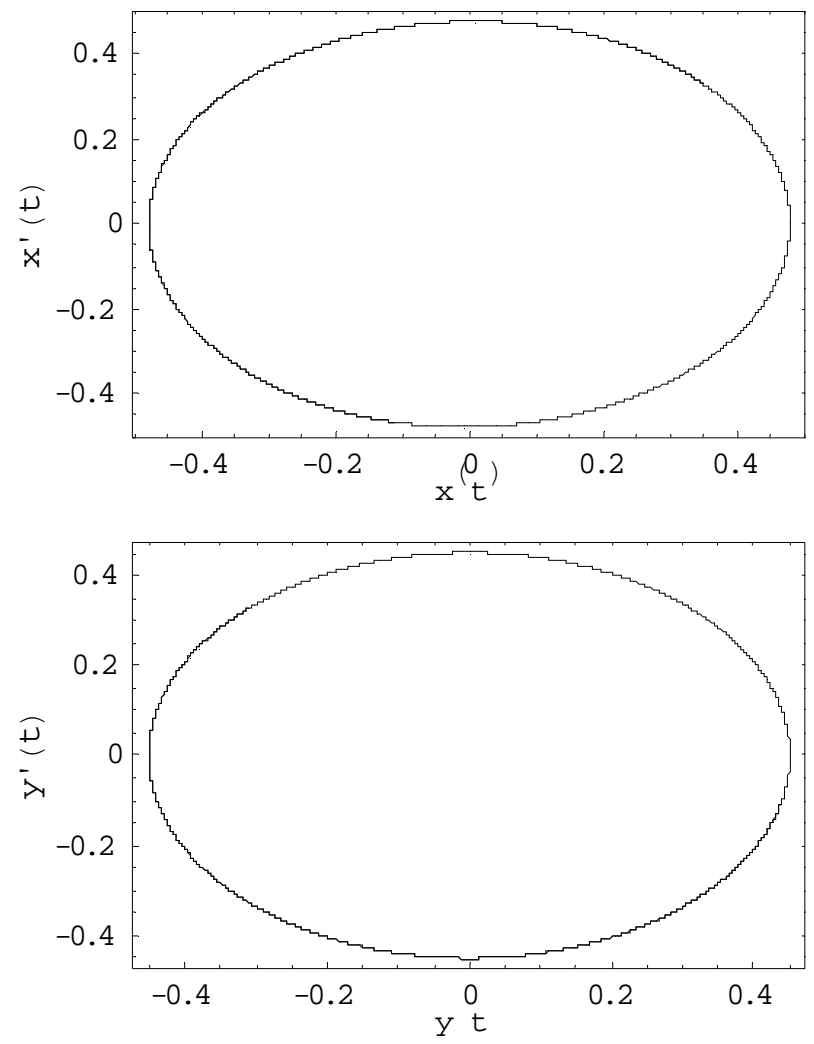

Fig. (2). Phase portraits of the limit cycle of equations (1)-(2) on the $x-x^{\prime}$ and $y-y^{\prime}$ planes when $\mu_{2}=0.7$.

$$
\begin{aligned}
& D_{0}^{2} y_{1}+\omega_{1}^{2} y_{1} \\
= & -2 D_{0} D_{1} y_{0}+\mu_{2} D_{0} y_{0}-c y_{0}^{3}+d x_{0},
\end{aligned}
$$

The solutions of equations (22)-(23) can be given by

$x_{0}=A \cos \left(T_{0}+\varphi_{1}\right)$ and $y_{0}=B \cos \left(T_{0}+\varphi_{2}\right)$,

where

$A=A\left(T_{1}\right), \varphi_{1}=\varphi_{1}\left(T_{1}\right), B=B\left(T_{1}\right), \varphi_{2}=\varphi_{2}\left(T_{1}\right)$,

which in fact means that $k=1$ in equation (17). Substituting equations (26) into the right-hand side of equations (24)-(25) yields

$$
\begin{aligned}
& D_{0}^{2} x_{1}+\omega_{1}^{2} x_{1}=\left(2 \omega_{1} D_{1} A-\mu_{1} \omega_{1} A+\frac{\mu_{1} \omega_{1} A^{3}}{4}\right) \sin \left(\omega_{1} T_{0}+\varphi_{1}\right) \\
& \quad+2 \omega_{1} A D_{1} \varphi_{1} \cos \left(\omega_{1} T_{0}+\varphi_{1}\right)+f \omega_{2}^{2} B \cos \left(\omega_{2} T_{0}+\varphi_{2}\right) \\
& \quad+\frac{3 \mu_{1} \omega_{1} A^{3}}{4} \sin \left(3 \omega_{1} T_{0}+3 \varphi_{1}\right) \\
& D_{0}^{2} y_{1}+\omega_{1}^{2} y_{1} \\
& =\left(2 \omega_{2} D_{1} B+\mu_{2} \omega_{2} B\right) \sin \left(\omega_{2} T_{0}+\varphi_{2}\right)
\end{aligned}
$$


$+\left(2 \omega_{2} B D_{1} \varphi_{2}-\frac{3 c B^{3}}{4}\right) \cos \left(\omega_{2} T_{0}+\varphi_{2}\right)$

$+d A \cos \left(\omega_{1} T_{0}+\varphi_{1}\right)-\frac{c B^{3}}{4} \sin \left(3 \omega_{2} T_{0}+3 \varphi_{2}\right)$.

Denote $v\left(T_{1}\right)=\varphi_{2}\left(T_{1}\right)-\varphi_{1}\left(T_{1}\right)$ and let us recall the fact of $\omega_{2}=\omega_{1}$. Consequently, $\cos \left(\omega_{2} T_{0}+\varphi_{2}\right)$ in equation (28) can be rewritten as $\cos \left(\omega_{1} T_{0}+\varphi_{1}+v\right)$ and similarly $\cos \left(\omega_{1} T_{0}+\varphi_{1}\right)$ in equation (29) can be rewritten as $\cos \left(\omega_{2} T_{0}+\varphi_{2}-v\right)$. Thus, elimination of the secular terms in equation (28)-(29) requires

$$
\left\{\begin{array}{l}
\left(2 \omega_{1} D_{1} A-\mu_{1} \omega_{1} A+\frac{\mu_{1} \omega_{1} A^{3}}{4}\right)-f \omega_{2}^{2} B \sin v=0, \\
\left(2 \omega_{2} D_{1} B+\mu_{2} \omega_{2} B\right)+d A \sin v=0 \\
2 \omega_{1} A D_{1} \varphi_{1}+f \omega_{2}^{2} B \cos v=0 \\
2 \omega_{2} B D_{1} \varphi_{2}-\frac{3 c B^{3}}{4}+d A \cos v=0
\end{array}\right.
$$

where $v\left(T_{1}\right)=\varphi_{2}\left(T_{1}\right)-\varphi_{1}\left(T_{1}\right)$.

Equations (30) can be transformed into

$$
\left\{\begin{array}{l}
D_{1} A=\frac{\mu_{1} A}{2}\left(1-\frac{A^{2}}{4}\right)+\frac{f \omega_{2}^{2} B}{2 \omega_{1}} \sin v, \\
D_{1} B=-\frac{\mu_{2} B}{2}-\frac{d A}{2 \omega_{2}} \sin v, \\
D_{1} v=-\frac{3 c B^{3}}{8 \omega_{2}}-\left(\frac{d A}{2 \omega_{2} B}-\frac{f \omega_{2}^{2} B}{2 \omega_{1} A}\right) \cos v .
\end{array}\right.
$$

Equations (31) are identical with that of Chedjou et al. [6] derived by the averaging method.

Due to the fact that limit cycles are the steady-state solutions of dynamical systems, the amplitude and frequency of the fundamental solution (26) of equations (1)-(2) can be solved from the following nonlinear algebraic equations by setting the right-hand side of equations (31) to zero.

$$
\left\{\begin{array}{l}
\frac{\mu_{1} A}{2}\left(1-\frac{A^{2}}{4}\right)+\frac{f \omega_{2}^{2} B}{2 \omega_{1}} \sin v=0, \\
-\frac{\mu_{2} B}{2}-\frac{d A}{2 \omega_{2}} \sin v=0, \\
-\frac{3 c B^{3}}{8 \omega_{2}}-\left(\frac{d A}{2 \omega_{2} B}-\frac{f \omega_{2}^{2} B}{2 \omega_{1} A}\right) \cos v=0 .
\end{array}\right.
$$

It can be deduced from equations (32) that

$$
\left\{\begin{array}{l}
B^{2}=\frac{d \omega_{1} \mu_{1} A^{2}\left(1-\frac{A^{2}}{4}\right)}{f \omega_{2}^{3} \mu_{2}} \\
\frac{2 c B^{3}}{8 \omega_{2}}=\frac{1}{2}\left(\frac{d A}{\omega_{2}}-\frac{f \omega_{2}^{2} B^{2}}{\omega_{1} A}\right) \sqrt{1-B^{2}\left(\frac{\mu_{2} \omega_{2}}{d A}\right)^{2}} .
\end{array}\right.
$$

Hence, $A$ and $B$ can be solved from equations (33). Till now, the first-order approximation for the limit cycle of equations (1)- (2) is given by

$$
\begin{aligned}
& x(t)=A \cos \left(t+\varphi_{1}\right)-\frac{\mu_{1} A^{3}}{32 \omega_{1}} \sin \left(3 \omega_{1}+3 \varphi_{1}\right), \\
& y(t)=B \cos \left(t+\varphi_{2}\right)-\frac{c B^{3}}{32 \omega_{2}^{2}} \cos \left(3 \omega_{2}+3 \varphi_{2}\right),
\end{aligned}
$$

where the constants $A$ and $B$ are determined by equations (33).

If the parameters $\mu_{1}=0.33, d=0.66, f=0.33, c=0.01$, and $\omega_{1}=\omega_{2}=1.0$ are fixed and $\mu_{2}=0.7$ is chosen, it can be deduced from equations (33) that $A=0.478152$ and $B=0.450826$. Fig. (3) shows the phase portraits of the limit cycle of equations (1)-(2) on the $x-x^{\prime}$ and $y-y^{\prime}$ planes, from which we know that the analytical and numerical integrated results fit very well.

With the control parameter $\mu_{2}$ increasing from the Hopf bifurcation point $\mu_{2 c}=0.66$, for example, when it reaches $\mu_{2}=2.0$, the phase portraits of the analytical and numerical integrated results are illustrated in Fig. (4). We find that on the $x-x^{\prime}$ plane, the analytical result agrees well with that of numerical integration. However, on the $y-y^{\prime}$ plane, the amplitude derived from numerical integration is larger than that of analytically approximate method. The reason for this phenomenon is clear if we note that the parameter $\mu_{2}$ is the damped parameter of equation (2).
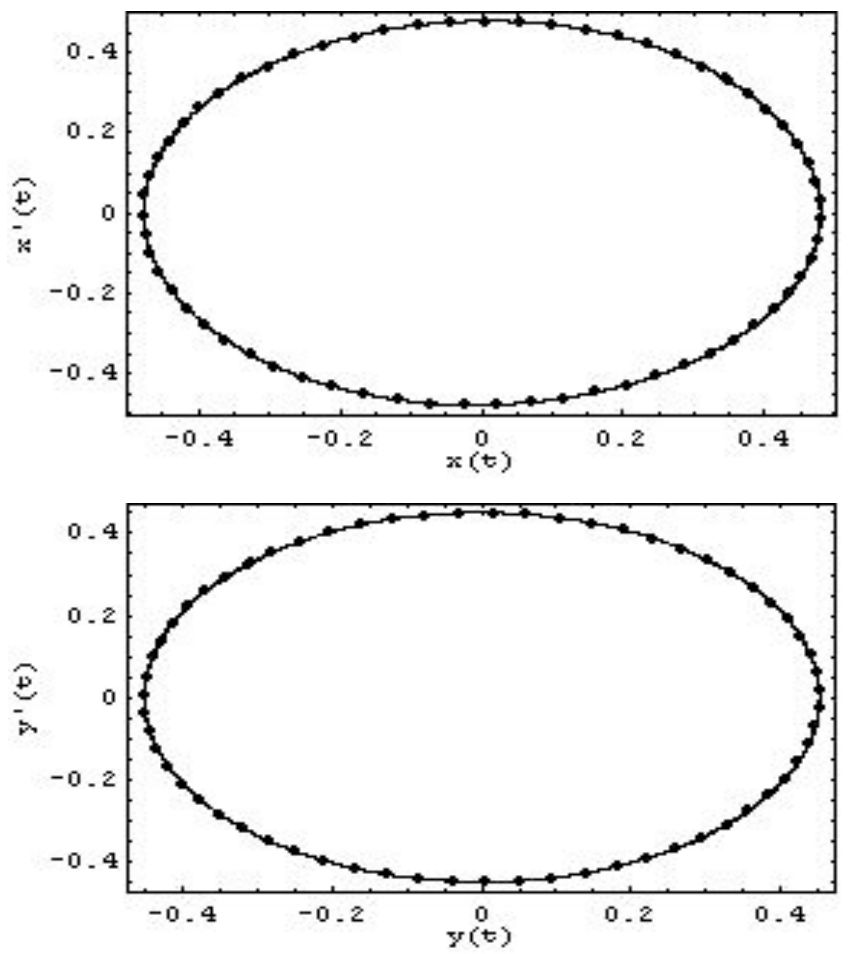

Fig. (3). Phase portraits of the limit cycle on the $x-x^{\prime}$ and $y-y^{\prime}$ planes when $\mu_{2}=0.7$, dot line for numerically integrated result and solid line for analytical result. 


\section{CONCLUSIONS}

Stability of the equilibrium point of coupled van der Pol and Duffing oscillators is investigated by using the RouthHurwitz criterion. Utilizing Hopf bifurcation theorem, existence of limit cycle is proved. The critical parametric curve corresponding to Hopf bifurcation and the existence region for the limit cycle in the parameter space are also obtained. The method of multiple scales is applied to deduce the analytical approximation of the limit cycle. If the control parameter is closed to the Hopf bifurcation point, the analytical and numerical integrated results are well agreed. However, as the control parameter increases gradually and deviates from the Hopf bifurcation point, the analytical approximations do not match well with numerical integrated results.
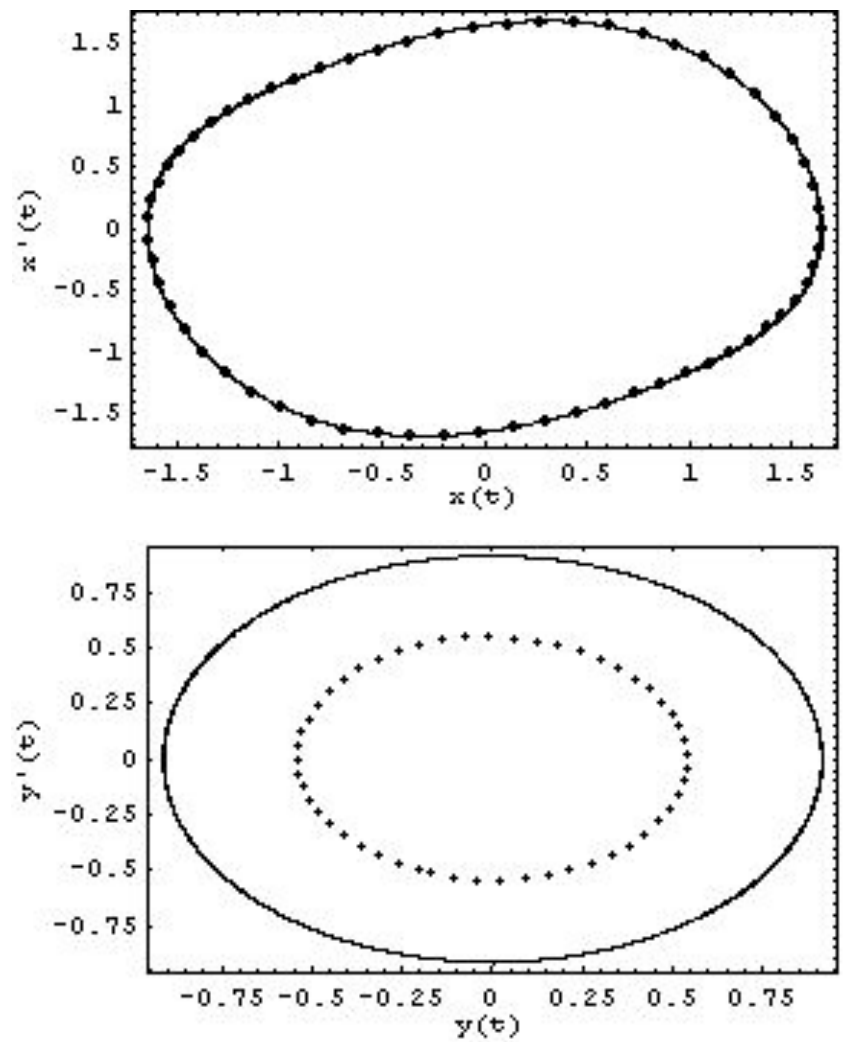

Fig. (4). Phase portraits of the limit cycle on the $x-x^{\prime}$ and $y-y^{\prime}$ planes when $\mu_{2}=2.0$, dot line for numerically integrated result and solid line for analytical result.

\section{ACKNOWLEDGEMENT}

Research is supported by the Foundation for Supporting Universities in Fujian, China under grant No 2007F5099.

\section{REFERENCES}

[1] Kozlowsky J, Parlitz U, Lauterborn W. Bifurcations analysis of two coupled periodically driven Duffing oscillators. Phys Rev E 1995 51: 1861-1867.

[2] Kenfack A. Bifurcation structure of two coupled periodically driven double-well Duffing oscillators. Chaos Solitons Fractal 2003; 15(2): 205-218.

[3] Pastor I, Lopez A. Dynamics of two coupled van der Pol oscillators. Phys Rev E 1995; 52: 1480-1489.

[4] Pastor I, Pérez-García VM, Encinas F, Guerra JM. Ordered and chaotic behavior of two coupled van der Pol oscillators. Phys Rev E 1993; 48: 171-182.

[5] Bi Q S. Dynamical analysis of two coupled parametrically excited van der Pol oscillators. Int J NonLinear Mech 2004; 39 (1): 33-54.

[6] Chedjou J C, Fotsin H B, Woafo P, Domngang S. Analog simulation of the dynamics of a van der Pol oscillator coupled to a Duffing oscillator. IEEE Trans Circuits Syst I Fundam Theor Appl 2001; 48(6): 478-757.

[7] Chedjou J C, Woafo P, Domngang S. Shilnikov chaos and dynamics of a self-sustained electromechanical transducer. ASME J Vib Acoust 2001; 123: 170-174.

[8] Hassard B D, Hazzarinoff N D, Wang Y H. Theory and application of Hopf bifurcation, Cambridge, 1981. 